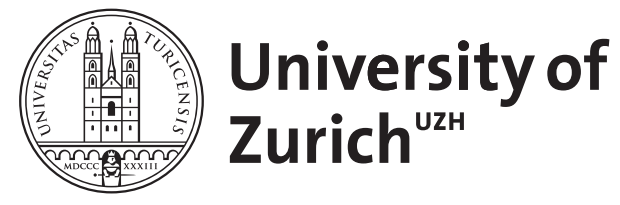
Archive

University of Zurich

Main Library

Strickhofstrasse 39

CH-8057 Zurich

www.zora.uzh.ch

Year: 2017

\title{
Prevalence and in-hospital outcome of aspiration in out-of-hospital intubated trauma patients
}

Radu, Raluca R; Kaserer, Alexander; Seifert, Burkhardt; Simmen, Hans-Peter; Ruetzler, Kurt; Spahn, Donat R; Neuhaus, Valentin

DOI: https://doi.org/10.1097/MEJ.0000000000000465

Posted at the Zurich Open Repository and Archive, University of Zurich

ZORA URL: https://doi.org/10.5167/uzh-136821

Veröffentlichte Version

Originally published at:

Radu, Raluca R; Kaserer, Alexander; Seifert, Burkhardt; Simmen, Hans-Peter; Ruetzler, Kurt; Spahn, Donat R; Neuhaus, Valentin (2017). Prevalence and in-hospital outcome of aspiration in out-of-hospital intubated trauma patients. European Journal of Emergency Medicine:Epub ahead of print.

DOI: https://doi.org/10.1097/MEJ.0000000000000465 


\section{Prevalence and in-hospital outcome of aspiration in out-of-hospital intubated trauma patients Raluca R. Radu ${ }^{a, \star}$, Alexander Kaserer ${ }^{a, \star}$, Burkhardt Seifert ${ }^{c}$, Hans-Peter Simmen ${ }^{b}$, Kurt Ruetzler ${ }^{a, d}$, Donat R. Spahn ${ }^{a}$ and Valentin Neuhaus ${ }^{b}$}

Objective The aim of this study was to evaluate the prevalence for aspiration in out-of-hospital intubated trauma patients and its impact on the in-hospital outcome.

Patients and methods Medical records of out-of-hospital intubated trauma patients, admitted from 2009 to 2014 to a level 1 trauma center, were retrospectively reviewed.

Patients younger than 18 years, treated with a supraglottic airway device, without a thorax computed tomography at admission, and with lung contusions were excluded. Two hundred and eighty-one patients were further analyzed. The definition of aspiration was based on computer tomographic findings at admission. Variables were analyzed in multivariate logistic and Cox regression analyses.

Results Aspiration occurred in 90 (32\%) patients. The mean Injury Severity Score was significantly higher in the aspiration group ( $40 \pm 26$ vs. $33 \pm 22, P=0.032$ ), whereas the mean initial Glasgow Coma Scale (GCS) on scene was significantly lower $(5 \pm 3$ vs. $7 \pm 4, P<0.001)$. In multivariate analysis, a lower initial GCS was a significant predictor for aspiration. Pneumonia, systemic inflammatory response syndrome, and sepsis occurred at similar frequencies. The 1 -day ( 41 vs. $23 \%, P=0.003$ ) and the 30 -day mortality (53 vs. $34 \%, P=0.003$ ) were significantly higher in the aspiration group. In survival analysis, a lower initial GCS, a higher

\section{Introduction}

Early intubation on scene in trauma patients is indicated according to international trauma guidelines including PHTLS, ATLS or ETC. Especially patients with traumatic head injury or shock benefit from early intubation and controlled ventilation [1-3]. Furthermore, intubation prevents pulmonary aspiration [4,5]. However, urgent intubation per se is also associated with an increased risk of aspiration [6,7]. Trauma patients are often not fasted; blood, vomitus, or swelling may make it difficult to visualize the larynx and vocal cords. In addition, difficult airway situations (face and neck trauma; constrained mouth opening; cervical spine immobilization), low Glasgow Coma Scale (GCS), obesity, difficult environmental conditions (poor light; limited space), as well as inexperienced physicians can increase the risk of aspiration [8]. Previous studies have reported incidences between 1 and $20 \%$ among various patient groups requiring emergent airway management in either the emergency department or the ICU [6,9]. In contrast, data on pulmonary aspiration in the out-of-hospital setting are
Injury Severity Score, and older age were independent predictors of 30-day mortality, but not aspiration.

Conclusion Aspiration was frequent in out-of-hospital intubated trauma patients and was associated with higher mortality and less favorable outcome, but was not an independent predictor of mortality and morbidity. However, a low initial GCS on scene was shown to be an independent predictor for aspiration and mortality. European Journal of Emergency Medicine 00:000-000 Copyright (C) Wolters Kluwer Health, Inc. All rights reserved.

European Journal of Emergency Medicine 2017, 00:000-000

Keywords: aspiration, intubation, outcome, out-of-hospital, prevalence, trauma

anstitute of Anesthesiology, ${ }^{\mathrm{b}}$ Department of Surgery, Division of Trauma Surgery, University Hospital Zurich, 'Division of Biostatistics at Epidemiology, Biostatistics and Prevention Institute, University of Zurich, Zurich, Switzerland and ${ }^{\mathrm{d}}$ Departments of Outcome Research and General Anesthesiology, Anesthesiology Institute, Cleveland Clinic, Cleveland, Ohio, USA

Correspondence to Valentin Neuhaus, MD, PD, Department of Surgery, Division of Trauma Surgery, University Hospital Zurich, Raemistrasse 100, 8091 Zurich, Switzerland Tel: + 414425511 11; fax: + 414425544 06;

e-mail: valentin.neuhaus@gmx.ch

${ }^{*}$ Raluca R. Radu and Alexander Kaserer contributed equally to the writing of this article.

Received 14 November 2016 Accepted 6 March 2017

limited and the reported incidence ranges between 34 and $50 \%[6,7,10-12]$.

Possible complications after aspiration are airway obstruction, Mendelson's syndrome, pneumonia, or acute respiratory distress syndrome (ARDS), which are associated with prolonged ventilator weaning, ICU stay, and increased mortality [12-14].

The aim of this study was to evaluate the prevalence and to investigate the impact of aspiration on in-hospital outcome for out-of-hospital intubated trauma patients in a level 1 trauma center in Switzerland.

\section{Patients and methods}

In a retrospective study, all inpatient medical records from a level 1 trauma center in Switzerland between 2009 and 2014 were reviewed. The local ethical committee approved the study (KEK-ZH-No: 2011-0382).

In Switzerland, prehospital care in case of severely injured patients is usually provided by a medical doctor 
(in our area usually an anesthesia resident) and a paramedic team. Severely injured patients are transferred by ambulance or helicopter to one of 12 trauma centers. In our trauma center, a standardized clinical approach is provided in the resuscitation area, where severely injured patients are usually admitted first. The standard approach consists of a primary survey and further treatment according to ATLS or ETC is applied by the trauma staff including at least one senior and one junior anesthetist, one senior, and one junior trauma surgeon as well as several nurses. The whole-body computed tomography (CT) scan is considered the gold standard and is performed in all major trauma patients as soon as possible to evaluate their relevant injuries and determine further treatment.

All trauma patients who underwent out-of-hospital intubation during the defined study period were included $(n=434)$. Exclusion criteria were age younger than 18 years, supraglottic airway devices, no chest CT scan at admission, and lung contusions because of the difficulties in distinguishing contusions and aspiration on chest CT scan. A total of 281 trauma patients fulfilled the inclusion and exclusion criteria, were further analyzed, and divided into an aspiration group and a no-aspiration group. Junior and senior radiologists on call reviewed the CT scans. Classic radiographic findings in acute aspiration included ground glass opacity; perihilar, ill-defined, alveolar consolidations; multifocal patchy infiltrates; and segmental or lobar consolidation, which were usually localized to one or both lung bases. The definition of aspiration was therefore based on computer tomographic findings at admission.

We used International Classification of Diseases, 10threvision, German Modification $[15,16]$ codes to identify all injuries, chronic comorbidities, and acute adverse events. The International Classification of Diseases, 10th-revision0 codes were encoded by professional medical coders. The procedures were coded according to the catalogue of the Swiss Surgery Classification System [17]. The Injury Severity Score (ISS) was calculated by certified medical staff who successfully completed the Association for the Advancement of Automotive medicine Abbreviated Injury Scale course.

\section{Statistical analyses}

Categorical data were reported in absolute numbers and percent, and numerical data as mean \pm SD. The Pearson $\chi^{2}$ or Fisher's exact test was used to compare categorical data, whereas the Mann-Whitney $U$-test was used to compare numerical data. Explanatory variables included patient demographics (age and sex), comorbidities, and injuries (ISS and GCS). The first dependent end point (outcome) was the presence of aspiration. The secondary outcomes were 1-day as well as 30-day overall mortality. A stepwise backward multivariable logistic regression model was used to identify risk factors for aspiration and to compare aspiration, patient, and injury factors associated with mortality. Adjusted odds ratio with corresponding 95\% confidence interval (CI) were tabulated. Statistical significance was set as a two-tailed $P$-value of less than 0.05. To answer the question of whether aspiration is an independent risk factor for a negative inhospital outcome, time to mortality, time to death or complications, and time to extubation were included in survival Kaplan-Meier curves and compared with the log-rank Mantel-Cox test as well as in Cox regression analyses while controlling for aspiration, age, sex, ISS, GCS, and comorbidities. Two variables (liver failure and gastroesophageal reflux disease ) were excluded from the multivariate analyses as they were always associated with one of the possible outcomes. All statistical analyses were carried out by IBM SPSS Statistics 22 (SPSS Inc., Chicago, Illinois, USA).

\section{Results \\ Demographics, prevalence of aspiration, and risk factors for aspiration}

Ninety out of 281 (32\%) of our trauma patients admitted to our hospital had radiologic findings associated with pulmonary aspiration. Demographics such as age, sex, and source of admission were the same in both groups (Table 1). The mean ISS was significantly higher in the aspiration group ( $40 \pm 26$ vs. $33 \pm 22, P=0.032$ ), whereas the mean initial GCS on scene was significantly lower ( $5 \pm 3$ vs. $7 \pm 4, P<0.001)$. In terms of comorbidities, mental disorders were significantly less prevalent in the aspiration group ( 23 vs. $35 \%, P=0.048$ ), whereas cerebrovascular diseases were significantly more prevalent (13 vs. $5.2 \%, P=0.018)$; other comorbidities had similar frequencies in both groups (Table 2). A lower GCS was a significant risk factor for aspiration (odds ratio 0.84; $95 \%$ CI: $0.77-0.91 ; P<0.001)$ while controlling for age, sex, ISS, and comorbidities.

\section{In-hospital outcome}

Surgery related complications during the hospitalization, such as wound infection, dehiscence, and hematoma, occurred significantly less frequently in the aspiration group ( 3.3 vs. $10.0 \%, P=0.042)$. All other complications, in particular, pneumonia (20 vs. $17 \%, P=0.58$ ), systemic inflammatory response syndrome (4 vs. $6 \%, P=0.54$ ), and sepsis ( 4 vs. $4 \%, P=1.0$ ), occurred at similar frequencies. Length of mechanical ventilation was equal in both groups $(94 \pm 184$ vs. $90 \pm 146 \mathrm{~h}, P=0.43)$. The overall 1-day mortality ( 41 vs. $23 \%, P=0.003$ ) and the 30 -day mortality (53 vs. $34 \%, P=0.003$ ) were significantly higher in the aspiration group, whereas the length of in-hospital stay was significantly shorter $(9 \pm 13$ vs. $12 \pm 14$ days, $P=0.004$ ). 
Table 1 Overview

\begin{tabular}{|c|c|c|c|c|}
\hline Parameters & Total & No aspiration & Aspiration & $P$-value \\
\hline Patients & $281(100)$ & $191(68)$ & $90(32)$ & \\
\hline \multicolumn{5}{|l|}{ Sex } \\
\hline Female & $81(29)$ & $54(28)$ & $27(30)$ & 0.77 \\
\hline Male & $200(71)$ & $137(72)$ & $63(70)$ & \\
\hline Age (years) & $54 \pm 22$ & $53 \pm 21$ & $56 \pm 22$ & 0.23 \\
\hline \multicolumn{5}{|l|}{ Admission from } \\
\hline Private residence & 268 (95) & $185(97)$ & $83(92)$ & 0.08 \\
\hline Nursing home & $4(1.4)$ & $2(1.0)$ & $2(2.2)$ & 0.60 \\
\hline Other hospital & $7(2.5)$ & $3(1.6)$ & $4(4.4)$ & 0.22 \\
\hline Other & $2(0.7)$ & $1(0.5)$ & $1(1.1)$ & 0.54 \\
\hline Comorbidities & $160(57)$ & $116(61)$ & $44(49)$ & 0.61 \\
\hline Initial GCS on scene & $7 \pm 4$ & $7 \pm 4$ & $5 \pm 3$ & $<0.001$ \\
\hline ISS & $35 \pm 24$ & $33 \pm 22$ & $40 \pm 26$ & 0.032 \\
\hline Head injuries & $251(89)$ & $168(88)$ & $83(92)$ & 0.28 \\
\hline Extremities injuries & $129(46)$ & $98(51)$ & $31(34)$ & 0.008 \\
\hline Thoracic injuries & $106(38)$ & 75 (39) & $31(34)$ & 0.44 \\
\hline Spine injuries & 77 (27) & $57(30)$ & $20(22)$ & 0.18 \\
\hline Neck injuries & $44(16)$ & $35(18)$ & $9(10)$ & 0.073 \\
\hline Pelvic injuries & $43(15)$ & $33(17)$ & $10(11)$ & 0.18 \\
\hline Abdominal injuries & $40(14)$ & $30(16)$ & $10(11)$ & 0.30 \\
\hline $\begin{array}{l}\text { Length of hospital stay } \\
\text { (days) }\end{array}$ & $11 \pm 14$ & $12 \pm 14$ & $9 \pm 13$ & 0.004 \\
\hline Length of stay in ICU (h) & $125 \pm 193$ & $133 \pm 193$ & $110 \pm 193$ & 0.087 \\
\hline $\begin{array}{l}\text { Length of mechanical } \\
\text { ventilation }(h)\end{array}$ & $91 \pm 159$ & $90 \pm 146$ & $95 \pm 184$ & 0.43 \\
\hline $\begin{array}{l}\text { Any inpatient } \\
\text { complications (except } \\
\text { death) }\end{array}$ & 109 (39) & $83(44)$ & $26(29)$ & 0.019 \\
\hline 1-Day mortality & $81(29)$ & $44(23)$ & $37(41)$ & 0.003 \\
\hline 30-Day mortality & $113(40)$ & $65(34)$ & 48 (53) & 0.003 \\
\hline
\end{tabular}

Data are reported as frequency with $n(\%)$ or mean \pm SD. GCS, Glasgow Coma Scale; ISS, injury severity score.

Bold indicates significant $P$ values.

\section{Impact of aspiration on in-hospital outcome and other significant risk factors}

In survival analyses, aspiration resulted in significantly longer time to extubation, shorter time alive and free from complications as well as higher 30-day mortality (Figs 1-3). However, in multivariate analyses, aspiration was not an independent predictor for 30-day mortality, time to death, time to extubation or time alive, and free from complications while controlling for age, sex, ISS, GCS, and comorbidities.
Fig. 1

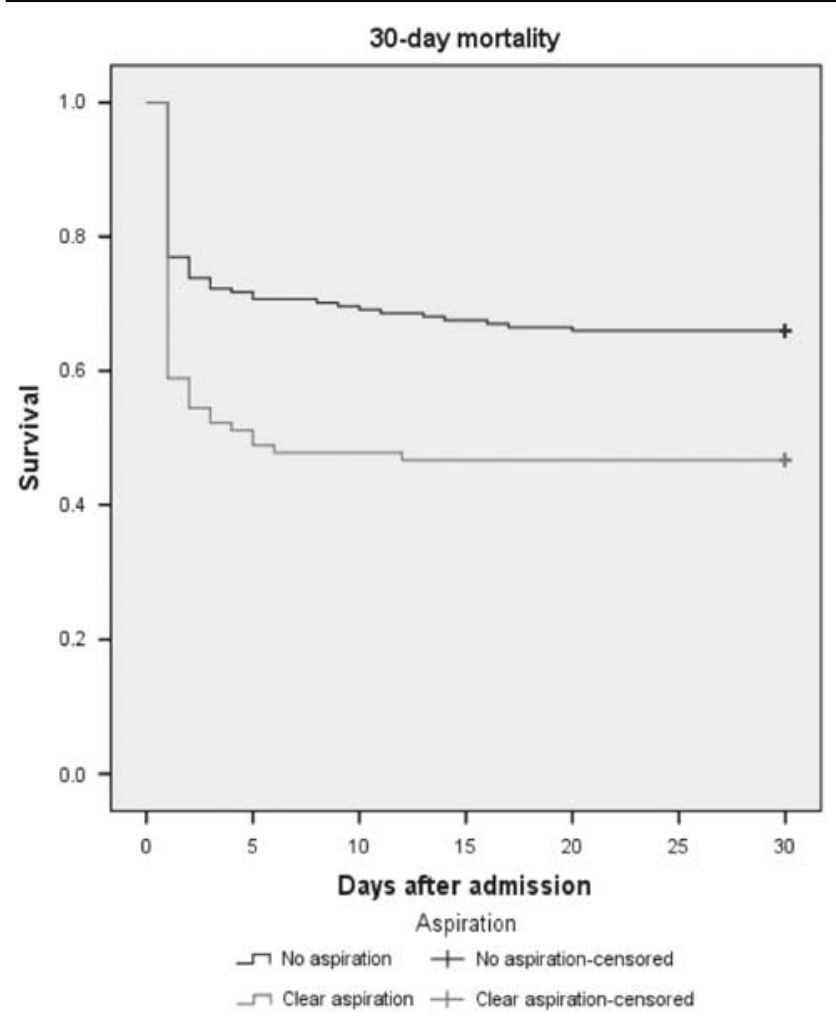

30-Day mortality: mortality in trauma patients with aspiration is significantly higher compared with patients without aspiration. $P=0.001$ (Mantel-Cox).

Older age, higher ISS, lower initial GCS, and inversely chronic alcoholism/mental disorders were independent predictors for 30-day mortality (Table 3) and shorter time to death. Higher ISS (HR: 1.013; 95\% CI: 1.007-1.020; $P<0.001)$ and older age (HR 1.008; 95\% CI 1.001-1.014; $P=0.016)$ were also predictors for longer time to extubation.

Table 2 Preaccident comorbidities

\begin{tabular}{|c|c|c|c|c|}
\hline Parameters & Total $[n(\%)]$ & No aspiration $[n(\%)]$ & Aspiration $[n(\%)]$ & $P$-value \\
\hline Mental disorders & $88(31)$ & $67(35)$ & $21(23)$ & 0.048 \\
\hline Chronic alcoholism & $24(9)$ & $18(9)$ & $6(7)$ & 0.44 \\
\hline Intoxication with psychotropic substances & $22(8)$ & $15(8)$ & 7 (8) & 0.98 \\
\hline Depression & $18(6)$ & $12(6)$ & $6(7)$ & 0.90 \\
\hline Hypertensive disease & $46(16)$ & $27(14)$ & $19(21)$ & 0.14 \\
\hline Arrythmias & $37(13)$ & $27(14)$ & $10(11)$ & 0.48 \\
\hline Atrial fibrillation & $21(8)$ & $15(8)$ & $6(7)$ & 0.72 \\
\hline Cerebrovascular disease & $22(8)$ & $10(5)$ & $12(13)$ & 0.018 \\
\hline Diabetes mellitus & $15(5)$ & $12(6)$ & $3(3)$ & 0.40 \\
\hline Esophagitis, gastroesophageal reflux disease, peptic ulcer disease, gastritis, duodenitis & $14(5)$ & $8(4)$ & $6(7)$ & 0.39 \\
\hline Muscle, skeleton and connective tissue disease & $11(4)$ & $10(5)$ & $1(1)$ & 1.00 \\
\hline Paraplegia or tetraplegia and hemiplegia & $8(3)$ & $5(3)$ & 3 (3) & 0.71 \\
\hline Malignancy & 7 (3) & $6(3)$ & $1(1)$ & 0.44 \\
\hline Chronic pulmonary disease & 7 (3) & 6 (3) & $1(1)$ & 0.44 \\
\hline Chronic coronary heart disease & $6(2)$ & $6(3)$ & $0(0)$ & 0.18 \\
\hline Congestive heart failure & $5(2)$ & $4(2)$ & $1(1)$ & 1.00 \\
\hline
\end{tabular}

Bold indicates significant $P$ values. 
Fig. 2

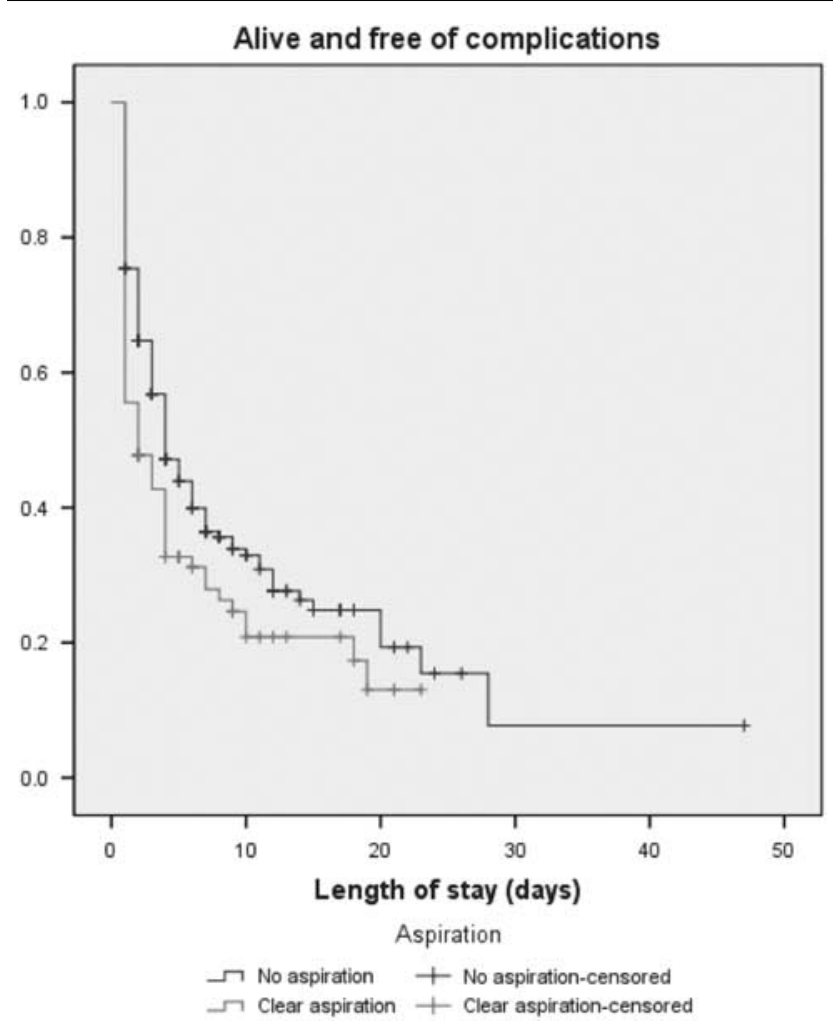

Alive and free of complications: the time alive and free of complications is significantly shorter in patients with aspiration. Patients who died were right censored from further analysis, depicted by a vertical bar on each graph. $P=0.028$ (Mantel-Cox).

\section{Discussion}

Aspiration is a common problem in the out-of-hospital airway management in trauma patients. Our study found an aspiration rate of $32 \%$ in out-of-hospital intubated trauma patients, which is slightly below the reported range from 34 to $50 \%$ [6,7,10-12]. Nevertheless, aspiration was not an independent predictor of negative inhospital outcome.

One-third of all intubated patients showed signs of aspiration on chest CT at admission in our study. These patients with aspiration had a significantly lower initial GCS on scene, which was shown to be an independent predictor in multivariate analysis. Aspiration is commonly found to be a frequent complication of rapid sequence intubation, although particularly a longer time in a low GCS state could increase the risk of aspiration as the ability to protect the airway from aspiration decreases with the GCS. It was shown that the majority of those trauma patients who aspirated did so before the intubation because of the decreased ability to protect the airway from aspiration [12]. In addition, we carried out a subanalysis excluding all patients with severe head injury; these results suggested that aspiration is rather the
Fig. 3

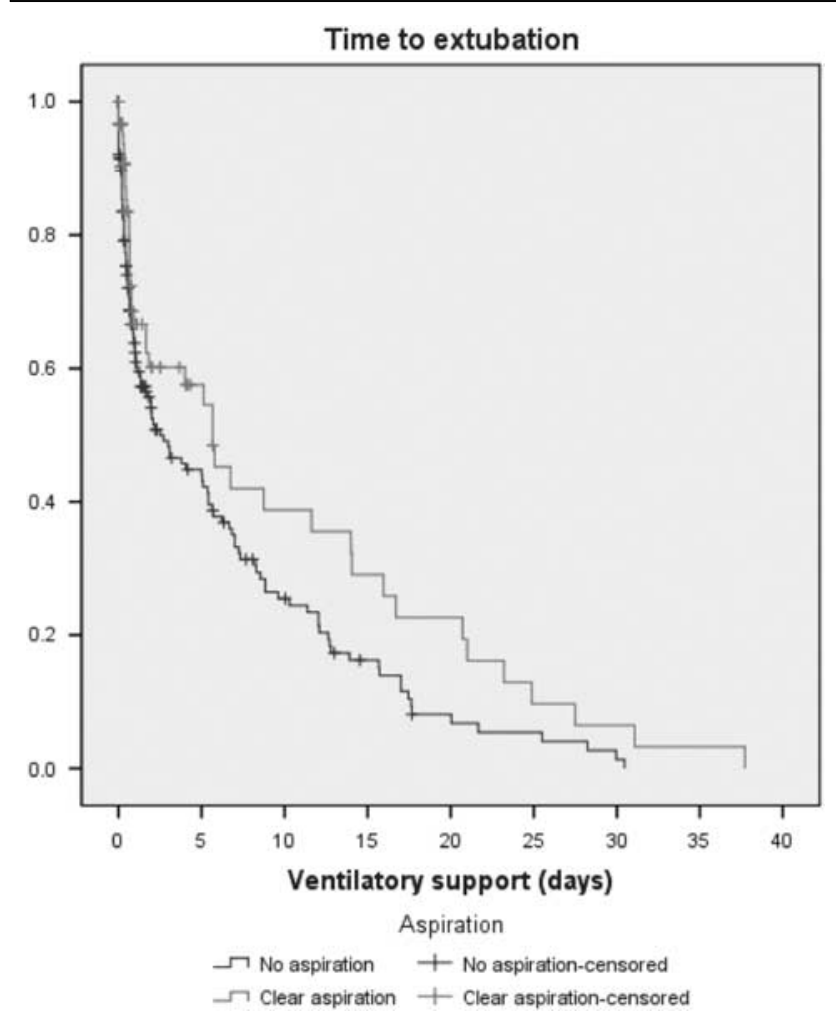

Time to extubation: trauma patients with aspiration were intubated and ventilated for a significantly longer duration. Patients who died were right censored from further analysis, depicted by a vertical bar on each graph. $P=0.028$ (Mantel-Cox).

Table 3 Independent 30-day mortality predictors

\begin{tabular}{llllr}
\hline & & \multicolumn{2}{c}{$95 \% \mathrm{Cl}$} & \\
\cline { 3 - 4 } & OR & Lower & Upper & $P$-value \\
\hline ISS & 1.118 & 1.083 & 1.154 & $<\mathbf{0 . 0 0 1}$ \\
Age & 1.033 & 1.014 & 1.052 & $\mathbf{0 . 0 0 1}$ \\
Initial GCS on scene & 0.74 & 0.64 & 0.85 & $<\mathbf{0 . 0 0 1}$ \\
Chronic alcoholism & 0.03 & 0.00 & 0.76 & $\mathbf{0 . 0 3 3}$ \\
\hline
\end{tabular}

Variables entered: age, sex, ISS, GCS, aspiration, intoxication with psychotropic substances, chronic alcoholism, depression.

$\mathrm{Cl}$, confidence interval; GCS, Glasgow Coma Scale; ISS, injury severity score; OR, odds ratio.

Bold indicates significant $P$ values.

consequence of a low GCS on scene than a complication because of the intubation.

Our study cohort had a high morbidity and 30-day mortality: both $40 \%$. The high mortality rate was because of our inclusion criteria. However, higher ISS, lower GCS, and higher age were comprehensible risk factors for this adverse outcome. Mental disorders and chronic alcoholism were associated with a more favorable outcome in terms of 30-day mortality and time to death. The assessment of a proper GCS in patients with mental disorders or intoxication is challenging. In our opinion, 
the initial GCS were probably lower scored in these patients by paramedics and physicians, which led to a liberal intubation strategy, although most probably indicated. The significant lower surgery-related complication rate and the shorter hospital stay in the aspiration group were rather caused by the higher mortality rate of these patients, especially in the first $24 \mathrm{~h}$ after admission. The higher mortality in the aspiration group might also have decreased the incidence of pneumonia in our study [18]. Pneumonia was still comparable in both groups. To the best of our knowledge, no comparable data on the impact of out-of-hospital aspiration in pneumonia have been published so far. We only found a controversial discussion on the impact on ventilator-associated pneumonia (VAP). Evans et al. [18] reported that out-of-hospital intubation in trauma patients was not associated with a higher risk of VAP. However, other studies have shown that rates of VAP are significantly higher (16-31\%) among patients intubated in an emergency situation following trauma $[12,19]$.

The fact that no significant increase in complications was observed in the aspiration group may also be the consequence of the current treatment strategy of these patients. Early, empiric, antibiotic therapy, controlled lung protective ventilation, and special surveillance in the intensive care unit might be a successful strategy to control typical aspiration-related complications such as pneumonia, sepsis, or ARDS. Nevertheless, aspiration is rather the consequence of a low GCS than a complication to the intubation and early, prehospital airway securing should not be under-rated as it is the first step in the prevention of aspiration and its related complications.

This study has several limitations that may have interfered with our results. First, this is a retrospective study and involves some variables that we will discuss further. Aspiration was diagnosed by the initial CT chest at admission; therefore, we have no information on the type of aspiration (blood, gastric contents, saliva, etc.) that was shown to affect pulmonary complications such as pneumonia, Mendelson syndrome, or ARDS [20]. We cannot conclude at which point the aspiration occurred: whether it was before, during, or after the intubation. In addition, we had no information on the use of drugs such as relaxants, hypnotics, and/or analgesics for rapid sequence induction and also for the use of antibiotics and their influence on in-hospital outcome. Another limitation is the out-of-hospital subjective estimation and evaluation of the patients such as GCS on scene, which led to the decision to intubation. Last but not the least, our patient population represents only traumatic injured patients from a single medical center and does not include any internal medicine patients.

\section{Conclusion}

Aspiration was present in one of three patients; it was associated with a higher 30-day mortality and less favorable outcome, but was not an independent predictor for mortality, time to extubation, complication, or time alive and free from complications. The current treatment strategy of trauma patients may address aspiration-related complications already in advance. However, a low initial GCS on scene was shown to be an independent predictor for aspiration and 30-day mortality.

\section{Acknowledgements}

Authors contribution: Raluca R. Radu and Alexander Kaserer contributed to data collection, data interpretation, drafting the manuscript, and critical revision of the manuscript. Burkhardt Seifert contributed to the statistical analysis of the manuscript. Hans P. Simmen contributed to critical revision of the manuscript. Kurt Ruetzler conceived and designed the study, contributed to data interpretation, and helped to draft the manuscript. Donat R. Spahn contributed to data interpretation, drafting the manuscript, and critical revision of the manuscript. Valentin Neuhaus participated in the design and coordination of the study, carried out the statistical analysis, contributed to data interpretation, drafting the manuscript, and critical revision of the manuscript. All authors read and approved the final manuscript.

\section{Conflicts of interest}

Dr Spahn's academic department is/has been receiving grant support from the Swiss National Science Foundation, Berne, Switzerland, the Ministry of Health (Gesundheitsdirektion) of the Canton of Zurich, Switzerland for Highly Specialized Medicine, the Swiss Society of Anesthesiology and Reanimation (SGAR), Berne, Switzerland, the Swiss Foundation for Anesthesia Research, Zurich, Switzerland, Bundesprogramm Chancengleichheit, Berne, Switzerland, CSL Behring, Berne, Switzerland, Vifor SA, Villars-sur-Glâne, Switzerland. Dr Spahn was the chair of the ABC Faculty and is the cochair of the ABC-Trauma Faculty, managed by Physicians World Europe GmbH, Mannheim, Germany and sponsored by unrestricted educational grants from Novo Nordisk HealthCare AG, Zurich, Switzerland, CSL Behring GmbH, Marburg, Germany and LFB Biomédicaments, Courtaboeuf Cedex, France. In the past 5 years, Dr Spahn has received honoraria or travel support for consulting or lecturing from: Abbott AG, Baar, Switzerland, AMGEN GmbH, Munich, Germany, AstraZeneca AG, Zug, Switzerland, Baxter AG, Volketswil, Switzerland, Baxter S.p.A., Roma, Italy, Bayer, Zürich, Switzerland and Berlin, Germany, B. Braun Melsungen AG, Melsungen, Germany, Boehringer Ingelheim (Schweiz) GmbH, Basel, Switzerland, BristolMyers-Squibb, Rueil-Malmaison Cedex, France and Baar, Switzerland, CSL Behring GmbH, Hattersheim am Main, Germany and Berne, Switzerland, Curacyte AG, Munich, Germany, Daiichi Sankyo (Schweiz) AG, Thalwil, Switzerland, Ethicon Biosurgery, Sommerville, New Jersey, USA, Fresenius SE, Bad Homburg v.d.H., Germany, 
Galenica AG, Bern, Switzerland (including Vifor SA, Villarssur-Glâne, Switzerland), GlaxoSmithKline GmbH \& Co. KG, Hamburg, Germany, Janssen-Cilag, Baar, Switzerland and Beerse, Belgium, LFB Biomédicaments, Courtaboeuf Cedex, France, Merck Sharp \& Dohme AG, Luzern, Switzerland, Novo Nordisk A/S, Bagsvärd, Denmark, Octapharma AG, Lachen, Switzerland, Organon AG, Pfäffikon/SZ, Switzerland, PAION Deutschland GmbH, Aachen, Germany, Pharmacosmos A/S, Holbaek, Denmark, Photonics Healthcare B.V., Utrecht, Netherlands, ratiopharm Arzneimittel Vertriebs-GmbH, Vienna, Austria, Roche, Reinach, Switzerland, Sarstedt AG \& Co., Nümbrecht, Germany, Schering-Plough International, Inc., Kenilworth, New Jersey, USA, Tem International GmbH, Munich, Germany, Verum Diagnostica GmbH, Munich, Germany, Vifor Pharma, Munich, Germany, Vienna, Austria and St. Gallen, Switzerland. For the remaining authors there are no conflicts of interest.

\section{References}

1 Donaubauer B, Fakler J, Gries A, Kaisers UX, Josten C, Bernhard M. Interdisciplinary management of trauma patients: update 3 years after implementation of the S3 guidelines on treatment of patients with severe and multiple injuries. Anaesthesist 2014; 63:852-864.

2 Bouillon B, Probst C, Maegele M, Wafaisade A, Helm P, Mutschler M, et al. Emergency room management of multiple trauma: ATLS(R) and S3 guidelines. Chirurg 2013; 84:745-752.

3 Langeron O, Birenbaum A, Amour J. Airway management in trauma. Minerva Anestesiol 2009; 75:307-311.

4 Vadeboncoeur TF, Davis DP, Ochs M, Poste JC, Hoyt DB, Vilke GM. The ability of paramedics to predict aspiration in patients undergoing prehospital rapid sequence intubation. J Emerg Med 2006; 30:131-136.

5 Piegeler T, Roessler B, Goliasch G, Fischer H, Schlaepfer M, Lang S, et al. Evaluation of six different airway devices regarding regurgitation and pulmonary aspiration during cardio-pulmonary resuscitation (CPR) - a human cadaver pilot study. Resuscitation 2016; 102:70-74.

6 Thibodeau LG, Verdile VP, Bartfield JM. Incidence of aspiration after urgent intubation. Am J Emerg Med 1997; 15:562-565.
7 Ufberg JW, Bushra JS, Karras DJ, Satz WA, Kueppers F. Aspiration of gastric contents: association with prehospital intubation. Am J Emerg Med 2005; 23:379-382.

8 Thierbach A, Piepho T, Wolcke B, Kuster S, Dick W. Prehospital emergency airway management procedures. Success rates and complications. Anaesthesist 2004; 53:543-550.

9 Schwartz DE, Matthay MA, Cohen NH. Death and other complications of emergency airway management in critically ill adults. A prospective investigation of 297 tracheal intubations. Anesthesiology 1995; 82:367-376.

10 Lockey DJ, Coats T, Parr MJ. Aspiration in severe trauma: a prospective study. Anaesthesia 1999; 54:1097-1098.

11 Oswalt JL, Hedges JR, Soifer BE, Lowe DK. Analysis of trauma intubations. Am J Emerg Med 1992; 10:511-514.

12 Fawcett VJ, Warner KJ, Cuschieri J, Copass M, Grabinsky A, Kwok H, et al. Pre-hospital aspiration is associated with increased pulmonary complications. Surg Infect (Larchmt) 2015; 16:159-164.

13 Studer P, Raber G, Ott D, Candinas D, Schnuriger B. Risk factors for fatal outcome in surgical patients with postoperative aspiration pneumonia. Int $\mathrm{J}$ Surg 2016; 27:21-25.

14 Decelle L, Thys F, Zech F, Verschuren F. Ventilation-associated pneumonia after intubation in the prehospital or the emergency unit. Eur J Emerg Med 2013; 20:61-63.

15 Swiss Statistics. 14-Internationale Klassifikation der Krankheiten (ICD-10). ICD-10-GM Version 2010. Available at: http://www.dimdi.de/static/de/ klassilicd-10-gm/kodesuche/onlinefassungen/htm/gm2010/index.htm. [Accessed 7 July 2015].

16 World Health Organization (WHO). International statistical classification of diseases and related health problems (ICD-10). Available at: http://www. who.int/classifications/icd//CD10Volume2_en_2010.pdf. [Accessed 7 July 2015].

17 Swiss Statistics. 14-Schweizerische operationsklassifikation (CHOP). CHOP 2015. Available at: https://www.bfs.admin.ch/bfs/de/home/ statistiken/gesundheit/nomenklaturen/medkk/instrumente-medizinischekodierung.assetdetail.349295.html. [Accessed 7 July 2015]

18 Evans HL, Zonies DH, Warner KJ, Bulger EM, Sharar SR, Maier RV, et al. Timing of intubation and ventilator-associated pneumonia following injury. Arch Surg 2010; 145:1041-1046.

19 Evans HL, Warner K, Bulger EM, Sharar SR, Maier RV, Cuschieri J. Prehospital intubation factors and pneumonia in trauma patients. Surg Infect (Larchmt) 2011; 12:339-344.

20 Uchida K. Pathophysiology and therapeutic approach of pulmonary aspiration. Masui 2016; 65:13-22. 